\title{
Jungfrau detector for brighter X-ray sources - MX opportunities and IT challenges
}

Filip Leonarski ${ }^{a}$, Aldo Mozzanica a , Martin Brückner ${ }^{a}$, Carlos Lopez-Cuenca ${ }^{a}$, Sophie Redford ${ }^{a}$, Leonardo Sala ${ }^{a}$, Andrej Babic ${ }^{a}$, Heinrich Billicha, Oliver Bunk ${ }^{a}$, Bernd Schmitt ${ }^{a}$ and Meitian Wang ${ }^{\mathrm{a}}$

aPaul Scherrer Institut, Forschungsstrasse 111, 5232 Villigen PSI, Switzerland（filip-karol.leonarski@psi.ch)

Developments in hybrid pixel X-ray detectors expand the horizon of macromolecular crystallography. This was witnessed by Swiss Light Source MX beamline users with Pilatus [1] and Eiger [2] and will happen shortly with Jungfrau [3,4]. The low-noise, high dynamic range, and high framerate of such detectors enable faster measurement, increase beamline throughput, and are also important for time resolved synchrotron serial crystallography. In addition, each generation of detectors increases the accuracy of measurements, which is critical in measuring small differences in intensities for experimental phasing with native elements. Designed primarily for $\mathrm{X}$-ray free-electron laser applications, the charge integrating, adaptive gain detector Jungfrau is already in user operation at the SwissFEL endstations. Jungfrau also has the potential to push both accuracy and throughput of MX experiments even further for synchrotron applications - allowing the full flux of diffraction limited storage rings to be used and unlocking the potential of long wavelength native-SAD phasing. Results from SLS X06A will be presented to demonstrate the advantages of Jungfrau for macromolecular crystallography.

However, as framerates and number of pixels increase, the IT infrastructure of a facility becomes critical for the detector integration. Increasing data rates, that could exceed $10 \mathrm{~GB} / \mathrm{s}$, are impractical to handle with commodity hardware - therefore either the size or the framerate of the detector would need to be compromised for operational reasons. To overcome this limitation, at PSI we are actively developing a data acquisition and processing system, benefiting from emerging architectures and their combination with in-hardware processing (IBM POWER9, GPU and FPGA). That will allow a 10 Mpixel Jungfrau detector to be operated at rates above $2 \mathrm{kHz}$ for routine user operation and pave the way for operating even larger format detectors at a $\mathrm{kHz}$ frame-rate. A successful solution for a Jungfrau $4 \mathrm{Mpixel}$ at $1.1 \mathrm{kHz}(9.2 \mathrm{~GB} / \mathrm{s})$ will be shown, and the current status and plans for a system capable of up to $50 \mathrm{~GB} / \mathrm{s}$ will be discussed.

\section{References}

[1] Müller, M. et al. Acta Cryst (2012), D68, 42-56

[2] Casanas, A. et al. Acta Cryst (2016), D72, 1036-1048

[3] Mozzanica, A. et al. Synchrotron Radiat News (2018), 31, 16-20

[4] Leonarski, F. et al. Nat Methods (2018), 15, 799-804 